\title{
Reproductive decisions after neonatal screening identifies cystic fibrosis
}

\author{
Tracy Dudding, Bridget Wilcken, Bronwyn Burgess, Judy Hambly, Gillian Turner
}

\begin{abstract}
Aims-To document the reproductive choices made by women in New South Wales, Australia, after neonatal screening has identified cystic fibrosis (CF).

Methods-A sample of women attending cystic fibrosis clinics in New South Wales who had a child (or children) diagnosed by neonatal screening between 1981 and 1996 were interviewed.

Results-Two thirds of the women chose to avoid having another child with $\mathrm{CF}$. The uptake of prenatal diagnosis was $66 \%$ in women who had a subsequent pregnancy; of these $69 \%$ terminated or would have terminated an affected fetus. Fifty nine per cent of the women who decided against a further pregnancy made this decision in order to avoid having another child with CF.

Conclusions-These data show that having a child with CF influenced subsequent reproductive choices. In addition to the medical advantages of an early diagnosis offered by neonatal screening, this also allows informed future reproductive decisions.
\end{abstract}

(Arch Dis Child Fetal Neonatal Ed 2000;82:F124-F127)

Keywords: cystic fibrosis; screening; reproductive choices; genetic counselling

Neonatal screening for cystic fibrosis (CF) was started in New South Wales in 1981. Since then a total of 468 children have been diagnosed in this way. ${ }^{1}$ The primary aim of screening is to identify and treat affected babies early, to improve morbidity and mortality. Neonatal screening provides an opportunity to prevent malnutrition in infants with $\mathrm{CF}^{2}$ It also reduces anxiety in parents, as well as the number of hospital admissions. ${ }^{3}$ Although there is some evidence of pulmonary benefit, the effect of neonatal screening on long term pulmonary function remains to be clearly established.

Screening also allows women to make early informed reproductive choices about future pregnancies. Previous studies assessing attitudes towards reproductive decisions in women who have a child with CF suggest that most would choose to avoid having a future

Table 1 Summary of previous studies assessing attitudes to prenatal diagnosis (PND) for cystic fibrosis

\begin{tabular}{llll}
\hline & $\begin{array}{l}\text { Percentage who would use } \\
\text { PND }\end{array}$ & $\begin{array}{l}\text { Percentage who would consider } \\
\text { termination of an affected pregnancy }\end{array}$ & $\begin{array}{l}\text { Percentage who may decide } \\
\text { against further children }\end{array}$ \\
\hline Evers-Kieiboom et al 1990 & $66 \%(32 / 49)$ & $45 \%(22 / 49)$ & $61 \%(30 / 49)$ \\
Watson et al 1992 & $74 \%(118 / 159)$ & $44 \%(70 / 160)$ & $64 \%(98 / 153)$ \\
Weitz et al 1992 & $77 \%(24 / 31)$ & $28 \%$ & - \\
\hline
\end{tabular}

affected child, either by not having further children or by terminating an affected pregnancy (table 1). ${ }^{4-6}$ The parents in these previous studies differ from the screening population in that the affected children were symptomatic and diagnosed clinically. It could be argued that the identification of an asymptomatic child might not influence subsequent reproductive choices.

This study aimed to document the actual reproductive choices made in a subsequent pregnancy after the birth of a child with CF identified by neonatal screening; and to determine whether these decisions were influenced by: (i) perceived current health of the child; (ii) perceived future health of the child; (iii) perceived emotional, financial, and workload burden of caring for a child with CF; (iv) number of previously well children; (v) genetic counselling; and (vi) socioeconomic factors.

\section{Methods}

The sample included 164 mothers who had a child (or children) diagnosed with CF by neonatal screening between 1981 and 1996. Subjects were recruited from the John Hunter Hospital in Newcastle (19 children), the New Children's Hospital (100), and Sydney Children's Hospital (45). One hundred and thirty seven mothers agreed to participate, a response rate of $84 \%$. Mothers were excluded if they had changed their partner $(n=9)$ or had an early miscarriage $(n=4)$. This left a total of 124 mothers (fig 1). Some characteristics of the study population are shown in table 2 . In addition to reproductive decisions, mothers were asked to rate the financial, workload, and emotional burden of caring for a child with CF on a scale of $1-10$, with 10 representing the heaviest burden. They were also asked to rate their child's current health and their perceived future quality of life for the child on a scale of $1-10$, with 10 rating excellent.

Data was analysed using SPSS 6.1.4. The significance of contingency tables was tested using a continuity corrected $\chi^{2}$ or an exact $\chi^{2}$ if any cells had less than five expected counts. Associations between variables were expressed using odds ratios, and their 95\% confidence intervals $(95 \% \mathrm{CI})$ were calculated.

The New Children's

Hospital, Westmead,

NSW Australia

B Wilcken

Correspondence to:

Dr Tracy Dudding

email: tdudding@mail.

newcastle.edu.au 
Table 2 Some characteristics of study population

\begin{tabular}{ll}
\hline Racial background & $(\mathrm{n}=123)$ \\
White caucasian & 111 \\
Mediterranean & 10 \\
Indian & 2 \\
Level of education & $(\mathrm{n}=123)$ \\
Completed some high school & 46 \\
Completed year 12 & 37 \\
Tertiary education & 40 \\
Number of children in the family & $(\mathrm{n}=124)$ \\
1 child & 19 \\
2 children & 63 \\
3 children & 27 \\
4 children & 9 \\
$>4$ children & 6 \\
Number of children with $C F$ & $(\mathrm{n}=124)$ \\
1 child & 112 \\
2 children & 12 \\
\hline
\end{tabular}

\section{Results}

Mothers who were planning more children but had not yet made a decision about prenatal diagnosis $(n=13)$, or were never planning any further children after the birth of this child $(\mathrm{n}=24)$ where excluded from the final analysis, leaving a total of 87 women who made a reproductive choice dependent on having a child with CF (fig 1). A total of 53/87 (61\%) have had more pregnancies, and $66 \%(35 / 53)$ of these women had prenatal testing for cystic fibrosis. Most of the women (24/35, 69\%) either decided to terminate or indicated that they would have terminated an affected fetus, and a further four were unsure what they would have done had the fetus been predicted affected. Of the 12 pregnancies with a predicted affected fetus, 10 were terminated and two continued.

Eighteen women (34\%) had further pregnancies and declined prenatal diagnosis. Twelve gave "morally against termination" as the reason; five were "willing to have another child" with cystic fibrosis, and prenatal diagnosis was unavailable for one couple.

Of the 58 women who were not planning further pregnancies, $34(59 \%)$ indicated that they would have planned more children if they had not had a child with CF. Of these women,
20 were morally against termination and not willing to have another child with $\mathrm{CF}$ and nine $(26 \%)$ had no time to care for another child. Prenatal diagnosis was unavailable for three couples and two gave a mixture of different reasons for deciding against further children. Overall, $67 \%$ (58/87) of couples who made a reproductive choice decided on a strategy which avoided having a further affected child (table 3).

The data were divided into separate groups for further analysis. There was no significant difference between the three sample populations in terms of the percentage of women who made a reproductive choice to avoid a further child with CF (67\% in Newcastle, $67 \%$ at the New Children's Hospital, and 65\% at Sydney Children's Hospital). The number of previously well children appeared to influence reproductive decisions, although this difference was not significant (table 3 ). If there were no previously well children, $57 \%$ of women made a reproductive choice to avoid having another child with CF. However, if there was one previously well child, or two or more previously well children, $70 \%$ and $84 \%$, respectively, made reproductive decisions to avoid having a further child with CF ( $p=0.081$; NS).

The current general health of the child was rated greater than or equal to 7 by $81 \%$ of mothers, and greater than or equal to 9 by $45 \%$ of mothers. Overall, perceived future quality of life was rated lower than current general health, with $72 \%$ rating the former greater than or equal to 7 and $22 \%$ rating it greater than or equal to 9 . Women rated the emotional burden of caring for a child higher compared with workload or financial burden; the average ratings were 9,8 , and 5 , respectively. The recurrence risk of having a child with CF was correctly given by $98 \%$ of women. Over half the women (59\%) knew their child's DNA test result, but only $52 \%$ had ever seen a medical geneticist or genetic counsellor. The decision

Total 124

More pregnancies

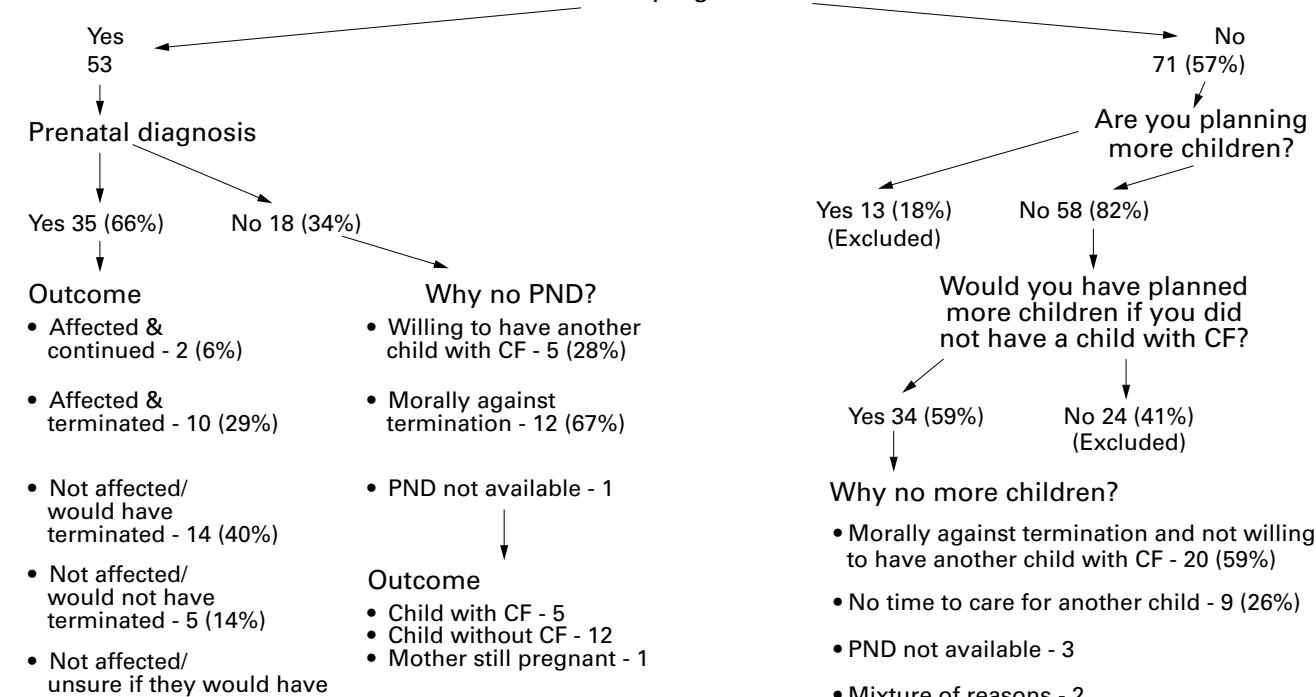

- Mixture of reasons - 2 
Table 3 Reproductive decisions based on the number of previously well children

\begin{tabular}{llll}
\hline & \multicolumn{2}{l}{ Number of previously well children } \\
\cline { 2 - 4 } & $0(n=40)$ & $1(n=34)$ & $2(n=13)$ \\
\hline Number that had more children & $31(77 \%)$ & $18(52 \%)$ & $4(30 \%)$ \\
Uptake of prenatal diagnosis & $22(70 \%)$ & $10(56 \%)$ & $3(75 \%)$ \\
Affected and terminated & $6 / 22$ & $4 / 10$ & $2 / 3$ \\
Unaffected and would have terminated & $8 / 22$ & $4 / 10$ & $1 / 3$ \\
Affected and continued & $2 / 22$ & $2 / 10$ & $9(69 \%)$ \\
Unaffected and would not have terminated & $4 / 22$ & $16(47 \%)$ & $9(13 \%)$ \\
Unaffected and unsure about termination & $9(23 \%)$ & $24 / 34(70 \%)$ & $11 / 13(84 \%)$ \\
Decision not to have further children dependent on having a child with CF & $23 / 40(57 \%)$ & & \\
Percentage who made a decision to avoid having another child with CF & &
\end{tabular}

to avoid having a further child with CF was not significantly influenced by the mother's level of education, the child's current health, perceived future quality of life, the perceived emotional, financial, or workload burden involved in caring for a child with $\mathrm{CF}$, or having received genetic counselling.

\section{Discussion}

The fact that we recruited mothers attending the CF clinic limits our study in some ways. For example, the absence of demographic data on the CF clinic families prevented comparison between respondents and non-respondents. Furthermore, families whose child had died from CF were excluded. Although the study addressed the reproductive choices made by couples, only the mothers were interviewed, thus leaving out the fathers' views.

The uptake of prenatal diagnosis and the percentage who decided against further children in our study were similar to results from previous studies where the diagnosis of CF was made clinically in a symptomatic child rather than by neonatal screening ${ }^{4-6}$ (table 1 ).

Our conclusions differ from those recently reported by Mischler et al. ${ }^{7}$ They concluded that neonatal screening does not have a significant impact on the reproductive behaviour of most families and that prenatal diagnosis is not used by most CF families. However, they have used only families where the child with CF was the first born to reach this conclusion, and have not taken into account the number of families who decided not to have further children because they had a child with CF. In 33 families where the child with $\mathrm{CF}$ was the first born child, $70 \%$ (23/33) conceived more children, but only $22 \%$ (5/23) utilised prenatal diagnosis. Of the 10 couples who decided against further children, $50 \%$ made this decision based on the fact that they had a previous child with CF. When the child with CF was the second or third born, $71 \%$ and $86 \%$ of couples, respectively, decided against further children. The authors do not state whether the decision not to have further children was influenced by the fact that they already had a child with CF.

Possible explanations for the lower uptake of prenatal diagnosis among women whose first child had CF in the study by Mischler et al compared with ours $(22 \%$ v $70 \%)$ include differences in genetic counselling or cultural attitudes to prenatal diagnosis and termination.

The issues arising around the benefits of early diagnosis through neonatal screening rather than a clinical diagnosis revolve around the effect of the lag period. Farrell et al reported a significant difference in the age of diagnosis, with a mean of 12 weeks by neonatal screening compared with 72 weeks in the clinically diagnosed group. ${ }^{2}$ The average age between a first and second child in Australia is 27.8 months; therefore, the average time from the birth of the first child to the next conception is 75 weeks. ${ }^{8}$ These data suggest that around $50 \%$ of subsequent children will be born before the clinical diagnosis of $\mathrm{CF}$ in the preceding child.

Antenatal screening for CF carrier status early in pregnancy is another screening programme which aims to offer couples the opportunity to make an informed reproductive choice. Although the numbers are small, Cunningham and Marshall ${ }^{9}$ reported a $65 \%$ reduction in the incidence of CF in Edinburgh during the first five years of antenatal screening (1991-95); the incidence of CF decreased from an average of 4.6 to 1.6 children per year. Only $36 \%$ of this reduction could be accounted for by prenatal diagnosis and termination, suggesting that couples may have decided not to have further children. Genetic testing was able to detect $72 \%$ of carrier couples, and $70 \%$ of couples agreed to be screened. This meant that only $50 \%$ of high risk pregnancies were identified. ${ }^{9}$

This study shows that neonatal screening provides the family with genetic information before the next pregnancy which alters their decision making. Overall, $67 \%$ of couples decided on a strategy which avoided having a further affected child, although one third had their reproductive confidence restored by the availability of prenatal diagnosis. Neonatal screening gives couples the opportunity of having more children without unknowingly taking the risk of having two affected children. This should be regarded as an additional benefit when reviewing national policy decisions about CF newborn screening.

We thank Dr Peter Cooper, Professor John Morton, and other respiratory physicians for permission to study the families of their patients; Ursula Bayliss, coordinator of the NSW Newborn Screening Program; Jenny Peat, Statistician at the New Children's Hospital; Sue Gilbert, CF nurse coordinator from Sydney Children's Hospital; Charlotte Wilson, CF nurse coordinator from the New Children's Hospital; and nurse coorRowley, Karla Tralor, Ann Hammond and Sharon Smith for their assistance.

1 Wilcken B, Wiley V, Sherry G, Bayliss U. Newborn screening for cystic fibrosis; a comparison of two strategies for case detection in 1.2 million babies. $f$ Pediatr 1995; 127:965-72.

2 Farrell PM, Kosorok MR, Laxova A, et al. Nutritional benefits of neonatal screening for cystic fibrosis. $N$ Engl f Med 1997;337:963-9. 
3 Wilcken B, Newborn screening for cystic fibrosis: its evolution and a review of the current situation. Screening $1983 \cdot 2 \cdot 463-8$

4 Evers-Kiebooms G, Denayer L, Van den Berge H. A child with cystic fibrosis: Subsequent family planning decisions, reproduction and use of prenatal diagnosis. Clin Genet 1990;37:207-15.

5 Watson EK, Marchant J, Bush A, Williamson B. Attitudes towards prenatal diagnosis and carrier screening for cystic fibrosis among the parents of patients in a paediatric cystic fibrosis clinic. f Med Genet 1992;29:490-1.

6 Wertz CD, Janes SR, Janet M, Rosenfield S, Erbe RW. Attitudes towards the prenatal diagnosis of cystic fibrosis: fac- tors in decision making among affected families. $A m \mathcal{F}$ Hum Genet 1992;50:1077-85.

7 Mischler EH, Wilfond BS, Fost N, et al. Cystic fibrosis newborn screening: impact on reproductive behavior and implications for genetic counselling. Pediatrics 1998;102:44-52.

8 Adam AY. Convergence on the two child family norm in Australia. Fournal of the Australian Population Association 1991;8:77-91.

9 Cunningham S, Marshall T. Influence of five years of antenatal screening on the paediatric cystic fibrosis population in one region. Arch Dis Child 1998;78:345-8. 